Bangladesh J Med Microbiol 2009; 03 (01): 39-43

Bangladesh Society of Medical Microbiologists

Mini Review

\title{
Laboratory Methods for Diagnosing Leptospirosis: A Review
}

\author{
Sofia Andalib Safiullah, ${ }^{1}$ Ahmed Abu Saleh, ${ }^{2}$ Shaila Munwar ${ }^{1}$ \\ ${ }^{1}$ Department of Microbiology, Medical College for Women and Hospital, Uttara, Dhaka, Bangladesh; ${ }^{2}$ Department of \\ Microbiology and Immunology, Bangabandhu Sheikh Mujib Medical University, Dhaka, Bangladesh
}

\begin{abstract}
Leptospirosis is a zoonotic disease that has been recognized as an important global health problem because of its increasing incidence in many countries and occurrence of several large outbreaks in recent years. Due to variability of clinical features and limited availability of laboratory facilities in endemic countries, the disease remains largely under-reported. Early and specific diagnosis is important to ensure a favourable outcome. Isolation of Leptospirosis is labourious and time-consuming. Direct demonstration of Leptospira from clinical specimens by dark-field microscopy (DFM), direct immunofluorescence, and immuno-peroxidase staining all lack specificity. Until now serological testing has been most frequently used to confirm diagnosis. Microscopic agglutination test (MAT) is the current gold standard but the technique is not simple and is only done in a few reference laboratories. To overcome these difficulties several test methods have been developed to detect IgM antibodies that are detectable after about 5th day of illness. In this paper, the microbiology, epidemiology and laboratory diagnosis of Leptospirosis have been discussed, and critically reviewed the advantages and disadvantages of various laboratory tests for the diagnosis of the disease.
\end{abstract}

\section{Introduction}

Leptospirosis is a spirochaetal zoonotic disease that has been recognized as an emerging infectious disease in the last 10 years. The disease has spread from its traditional rural base to become the cause of epidemics in poor urban slum communities in developing countries. ${ }^{1}$ The incidence of Leptospirosis is significantly higher in warm climate countries than in temperate regions. ${ }^{2,3}$ Although it has been suggested that Leptospirosis may be the commonest zoonotic disease, ${ }^{3}$ it has been greatly under reported due to nonspecificity of sign symptoms and limited availability of laboratory confirmation in endemic regions. ${ }^{4}$ Overall disease burden is underestimated as the disease has clinical features

\section{Correspondence:}

Dr. Sofia Andalib Safiullah

Associate Professor

Department of Microbiology

Medical College for Women and Hospital

Uttara, Dhaka, Bangladesh

E-mail: similar to many other febrile illnesses and there is a lack of simple, rapid tests, particularly in underdeveloped countries that hampers early management.

The purpose of this paper is to review the microbiology, epidemiology and laboratory diagnosis of leptospirosis.

\section{Microbiology}

Leptospires are thin tightly coiled spirochaetes with lengths of 6-20 $\mu \mathrm{m}$ and $0.01 \mu \mathrm{m}$ wide. The cells have pointed end, one or both ends of which are bent as hooks. They are too thin to be detected by ordinary microscopes, but can be visualized by dark field microscopy. The organism is motile. The motility is conferred by rotation of 2 axial flagella underlying the membrane sheath, which are inserted at opposite ends of the cells, and overlaps in the central region. Leptospires are obligate aerobes which derive energy by oxidation of long chain fatty acid. They do not use carbohydrate or amino acid as the major source of energy. 
Classification: The genus Leptospira was historically classified into 2 species $L$. interrogans and L. biflexa comprised of pathogenic and non-pathogenic strains respectively. Leptospires are now classified into a number of species defined by their degree of genetic relatedness, determined by DNA re-association. There are currently, 13 named species and four unnamed genomespecies. These include both pathogens and non-pathogens.

Table 1: Species of Leptospira and some pathogenic serovars

\begin{tabular}{ll}
\hline Species & Selected Pathogenic Serovars \\
\hline L. interrogans & Icterohaemorrhagiae, Copenhageni, Canicola, Pomona, \\
& Australis, Autumnalis, Pyogenes, Bratislava, Lai \\
L. noguchii & Pnama, Pomona \\
L. borgpetersenii & Ballum, Hardjo, Javanica \\
L. santarosi & Bataviae \\
L. kirscneri & Bim, Bulgarica, Grippotyphosa, Cynopteri \\
L. weilii & Celledoni, Sarmin \\
L. alexanderi & Manhao 3 \\
L. genomespecies 1 & Sichuan \\
L. fainei & Hurtsbridge \\
L. meyeri & Sofia \\
L. inadai & Indeterminate \\
\hline
\end{tabular}

\section{Epidemiology}

Leptospirosis is endemic throughout the world. Peak incidence occur in rainy seasons in tropical regions and the late summer to early fall in temperate climates. Outbreaks follow excessive rainfall.

Leptospirosis is maintained in nature by chronic renal infection of carrier animals. Most important reservoirs are rodents and other small mammals (e.g., mice, voles, hedgehogs), but livestock and companion animals are also significant sources of human infection. Infections of carrier animals occur in infancy. Once infected, they excrete leptospires intermittently or continuously throughout entire life.

Mode of transmission: Leptospires can enter through abrasions in skin, intact mucous membrane (e.g., conjunctiva, oronasopharynx). Infection occurs through direct or indirect contact with urine or tissues of infected animals. Direct contact is important in transmission to veterinarians, butchers and other animal handlers. Indirect contact is more common and responsible for disease following exposure to wet soil or water.
Human-to-human transmission is rare and can occur through sexual contact, transplacentally, and via breast-milk. Urine from a patient suffering from Leptospirosis should be considered infectious.

It is not known precisely when leptospires appear in the blood after infection. It is possible that during incubation period, before the infected person becomes ill, leptospires may circulate in the blood and be transmitted via blood transfusion.

Pathogenesis: The mechanism of disease production is not well understood. Potential virulence factors include attachment, toxin production, immune mechanisms and surface proteins. A systemic vasculitis occurs, facilitating migration of spirochaetes into organs and tissues and accounting for broad spectrum of clinical illness. Immunemediated mechanisms have been postulated as one factor influencing severity.

\section{Laboratory Diagnosis}

Dark field microscopy

Leptospires are too thin and take up conventional stains too poorly to be observed under ordinary light microscope. They are observed as thin, coiled, rapidly moving microorganisms in fluids such as blood, urine, using dark field microscopy. It is useful for observing Leptospires in culture, particularly when they are present in a large number, and for observing agglutination in Microscopic agglutination test (MAT). It is technically demanding. Recognizing Leptospires is difficult particularly when only small number of the spirochaete is present. Artifacts such as fibrin threads in blood are easily mistaken. This method has both low sensitivity (40.2\%) and specificity $(61.5 \%) .^{5}$

\section{Staining}

Immunofluorescence, immunoperoxidase, and silver staining can be used to detect leptospires directly in clinical specimen. Fluorescent microscope and expertise is required for immunofluorescence stain. Immunoperoxidase stain is not available commercially. The sensitivity of this test is also low. 5

\section{Culture}

Leptospires can be isolated from blood, CSF, peritoneal dialysate fluid, urine during first 10 days of illness. Most suitable specimen is blood. Specimens collected aseptically 
must be processed as soon as possible.

One to two drops of blood should be inoculated directly into culture media at bedside. Common media used for isolation is Ellinghausen and Mc Cullough modified by Johnson and Harris - (EMJH Media). ${ }^{6}$ The EMJH Media is inoculated and incubated aerobically at 28-30 degrees Celsius. Rate of growth is slow (doubling time 6-8 hours). Culture media should be checked for growth of Leptospires at regular intervals for a period of 4 months. The Dark field microscopy (DFM) is used for this purpose. Isolated Leptospires are further identified to serovar level, either by traditional serological methods, or by molecular methods such as pulse field gel electrophoresis. Leptospires grow slowly, so culture does not contribute to rapid diagnosis in early phases of disease and by the time culture becomes positive, patient develops detectable antibody. Culture is, therefore, relatively an insensitive diagnostic method. Cultured Leptospires can be used as antigen for various serological methods. Isolated Leptospires can be typed to identify serovars. Typing is useful in the surveillance of local pathogenic serovars, the recognition of new patterns of disease presentation, assaying the effectiveness of intervention measures.

\section{Polymerase Chain Reaction (PCR)}

The PCR can detect Leptospiral DNA in clinical samples like serum, urine, aqueous humour. The PCR can rapidly confirm the diagnosis in early phase of the disease when bacteria may be present and before antibody titre is in detectable levels. But PCR is expensive and requires equipments, dedicated laboratory space and highly skilled personnel. It may give false positive results in presence of extraneous DNA. The PCR is most sensitive in initial disease phase but is less sensitive than the serological tests over the course of the disease.

\section{Animal Inoculation}

Golden hamsters are usually used for this test. Blood or other clinical material is injected into the animal's abdominal cavity. A sharp pipette is used to collect a specimen of fluid from the abdominal cavity at regular intervals and is examined by dark field microscopy for the presence of the Leptospires. The animal is also observed for signs of disease. This method is now rarely used, as in vitro culturing yields comparable results and avoids animal suffering.

\section{Antigen detection in urine}

Monoclonal antibody-based dot-ELISA (Enzyme Linked Immunosorbent Assay) for detection of Leptospiral antigen in urine has been developed. However, this has not been evaluated widely and is not available commercially. ${ }^{6}$

\section{Antibody detection methods}

The methods available for the direct detection of Leptospires are either slow or of limited reliability or complicated. As a consequence, serology is often the best diagnostic method. In reality, patients usually seek medical care when they have already been ill for a sufficiently long time to have produced detectable antibodies.

\section{Microscopic Agglutination Test (MAT)}

The MAT is considered the gold standard for serodiagnosis of Leptospirosis. It determines agglutinating antibodies in the serum of a patient by mixing it in various dilutions with live or killed formalinized Leptospires. Anti-Leptospiral antibody present in the serum causes Leptospires to stick together to form clumps. This agglutination is observed by using dark field microscopy. Antibodies can be either IgM or IgG. According to the taxonomic sub-committee on Leptospira, the end-point is defined as that dilution of serum which shows $50 \%$ agglutination leaving $50 \%$ free cells when compared with control culture diluted $1: 2$ in phosphate buffer saline. ${ }^{7}$

The MAT is very specific, but has the following disadvantages: (i) facilities for culturing and maintaining live Leptospires are needed; (ii) the method is technically demanding and time-consuming, particularly when the panel is large; (iii) antibodies may not be detectable when the causative strain is not represented in the panel or only low titre is found with a serovar that antigenically resembles the absent causative serovar (the finding of no titre or low titre in the MAT does not exclude Leptospirosis in these circumstances, it is never possible to be sure that the panel is complete since new unidentifiable Leptospires may cause disease and for this reason, it is advisable to include a genus specific screening test, such as an ELISA using a broadly reactive antigen); (iv) the MAT cannot be standardized because live Leptospires are used as antigen.

Since test results may vary slightly from day-to-day, paired sera samples are best examined together. A degree of agglutination can be achieved by using formalin preserved 
Leptospires as antigens, but this applies only when the same batch of antigen is used. Unfortunately, preserved antigens denatures after only a few days.

\section{Enzyme Linked Immuno Sorbent Assay (ELISA)}

Conventional microtitre plate ELISA and dot-ELISA can detect IgM-class antibody in the early phase of disease, 24-48 hours before it can be detected by MAT, so that current or recent infections may be indicated. ${ }^{8}$ Whereas, whenever no antibody is detected or low titre is found, a second sample should be examined for seroconversion or a significant rise in titre. The test (antigen) can be standardized and commercial kits are available so there is no need for facilities for the culture of Leptospires in local laboratories to provide antigen. Studies have directly compared ELISA with MAT and reported quite high sensitivity (88-100\%) and specificity (9398\%). ${ }^{8-12}$ Two studies, where both the dot-ELISA and microplate-ELISA were used, have shown dot-ELISA to be more sensitive. ${ }^{10,12}$

Rheumatoid factors are auto-antibodies, mainly of IgM class. The presence of non-specific IgM antibodies (RA Factor) can lead to false-positive results in the IgM assay. Therefore, it is necessary to pre-treat samples with RA factor absorbance prior to IgM detection.

The ELISA has the following disadvantages: (i) only a single antigen is used, namely the genus-specific antigen which is shared by pathogenic and saprophytic leptospires alike; (ii) since it is based on genus-specific antigen, the ELISA test does not give an indication of infecting serovar.

\section{Other tests}

In addition to MAT and ELISA, various other tests have been developed. These include:

(a) IHA (Indirect Haemagglutination Assay): a simple test used for screening but having low sensitivity on samples from acute infections;

(b) IFA (Indirect Fluorescent Antibody test): highly specific but needs fluorescence microscope and therefore, cannot be used as a screening test;

(c) Latex agglutination: this can be done on a slide;

(d) IgM dipstick; and

(e) Immunochromatography (ICT) for Leptospira IgG.

\section{Conclusion}

The Microscopic Agglutination Test (MAT) is the standard test for the diagnosis of Leptospirosis. However, it is a difficult test and is currently only available in some reference laboratories. Alternative ELISA tests are simple and rapid and compare very well with the gold standard MAT in terms of sensitivity and specificity, particularly the dot-ELISA. The few studies that have looked for Leptospirosis in Bangladesh have discovered that the Leptospiral spirochaete is a common cause of febrile illness. ${ }^{13,14}$ However, Leptospires are usually not considered as the possible cause for fever in clinical practice in Bangladesh, which is believed largely due to the non-availability of testing facilities for Leptospirosis in the country and the consequent nonreporting of the disease in the local literature. It is recommended that the simple and rapid ELISA-based tests for Leptospira should be made available in Bangladesh so that good and appropriate treatment can be provided for this common cause of fever. This is important as leptospirosis is a potentially serious illness, but which is treatable, particularly, if detected early.

\section{References}

1. McBride AJ, Athanazio DA, Reis MG, Ko AI. Leptospirosis. Curr Opin Infect Dis 2005; 18: 376-386.

2. Everard JD, Everard COR. Leptospirosis in the Caribbean. Rev Med Microbiol 1993; 4: 114-122.

3. World Health Organization. Leptospirosis worldwide, 1999. Wkly Epidemiol Rec 1999; 74: 237-242.

4. Laras K, Van CB, Bounlu K, Tien NT, Olson JG, Thongchanh S, et al. The importance of leptospirosis in Southeast Asia. Am J Trop Med Hyg 2002; 67: 278-286.

5. Mandell GL, Bennett JE, Dolin R. Mandell, Douglas and Bennett's Principle and Practice of Infectious Diseases, Sixth Edition. Philadelphia, USA: Elsevier Churchill Livingstone; 2005.

6. World Health Organization. Human Leptospirosis: Guidance for Diagnosis, Surveillance and Control. Geneva, Switzerland: WHO; 2003.

7. International Committee on Systematic Bacteriology, Subcommittee on the Taxonomy of Leptospira. Minutes of the meeting, 6 to 10 August, 1982, Boston, Massachusetts, USA. Int J System Bacteriol 1984; 34: 258-259.

8. Cumberland P, Everard CO, Levett PN. Assessment of the efficacy of an IgM-ELISA and microscopic agglutination test 
(MAT) in the diagnosis of acute leptospirosis. Am J Trop Med Hyg 1999; 61: 731-734.

9. Ooteman MC, Vago AR, Koury MC. Evaluation of MAT, IgM ELISA and PCR methods for the diagnosis of human leptospirosis. Microbiol Methods 2006; 65: 247-257.

10. Tansuphasiri U, Deepradit S, Phulsuksombati D, Tangkanakul W. A test strip IgM dot-ELISA assay using leptospiral antigen of endemic strains for serodiagnosis of acute leptospirosis. $\mathrm{J}$ Med Assoc Thai 2005; 88: 391-398.

11. Vitale G, La Russa C, Galioto A, Chifari N, Mocciaro C, Caruso R, et al. Evaluation of an IgM-ELISA test for the diagnosis of human leptospirosis. New Microbiol 2004; 27: 149-154.

12. Levett PN, Branch SL. Evaluation of two enzyme-linked immunosorbent assay methods for detection of immunoglobulin M antibodies in acute leptospirosis. Am J Trop Med Hyg 2002; 66: 745-748.

13. LaRocque RC, Breiman RF, Ari MD, Morey RE, Janan FA, Hayes JM, et al. Leptospirosis during dengue outbreak, Bangladesh. Emerg Infect Dis 2005; 11: 766-769.

14. Morshed MG, Konishi H, Terada Y, Arimitsu Y, Nakazawa T. Seroprevalence of leptospirosis in a rural flood prone district of Bangladesh. Epidemiol Infect 1994; 112: 527-531.

[ Conflict of Interest: none declared] 